\title{
An Analysis of the Effect of the Discontinuity in a Bifurcated Circular Guide Upon Plane Longitudinal Waves
}

\author{
Louis L. Bailin*
}

\begin{abstract}
The problem of theoretically accounting for the scattering of sound by a semi-infinite circular tube with a small diameter inserted axially, as a measuring probe, into a larger tube of infinite length, is solved in a rigorous and explicit manner when the incident plane waves are restricted to the lowest propagating mode.

The investigation follows the methods outlined by J. Schwinger and consists in solving Helmholtz's equation subject to boundary conditions on the acoustic velocity potential at rigid walls. With the aid of Green's theorem and the Green's function for a point source in the large guide, an integral representation of the desired velocity potential is obtained. The imposition of the boundary conditions leads directly to a homogeneous integral equation of the Wiener-Hopf type, which is solved by transform techniques. The results of this solution are shown to be related to a distance $d$, which is the length the inner guide must be extended to account for the disturbance occurring in the immediate vicinity of the discontinuity caused by the excitation of higher mode fields.

Another satisfactory method of representing the effect of the discontinuity is by the use of equivalent circuits, by means of which a discontinuity in a wave guide is replaced by a lumped parameter network in a set of transmission lines. The equivalent circuit for this network is derived, and the one essential circuit parameter is related to $d$ and to the explicit solution of the integral equation.

Numerical results for $d$ are given as a function of several different guide ratios and driving frequencies of the incident waves. The results are in considerable disagreement with approximation methods previously reported and indicate that these methods are unsatisfactory in the present problem.
\end{abstract}

\section{Introduction}

The problem of theoretically accounting for the scattering of sound by a circular tube with a small diameter inserted axially into a larger tube as a measuring probe (see fig. 1) is merely a specialization of a general class of problems dealing with the effects of obstacles on the propagating modes of both acoustic and electromagnetic waves in guides. It has been shown by J. Schwinger [1] ${ }^{2}$, in a series of theoretical studies, that the special class of boundary-value

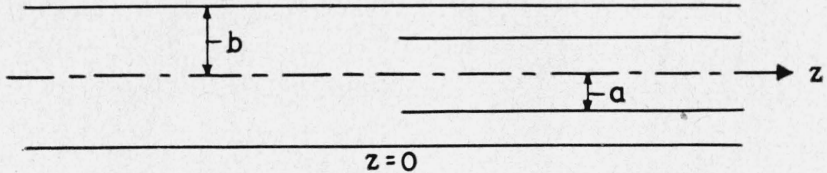

Figure 1.

problems, where waves are incident upon a number of semi-infinite parallel metallic structures (structures with parallel axes) of zero thickness and perfect conductivity, can be formulated mathematically as an inhomogeneous integral equation of the Wiener-Hopf type [2]. Because of the discontinuity, the imposition of the particular boundary conditions that the field components must satisfy leads directly to one or more intregral equations of the type

$$
g(x)=\int_{0}^{\infty} K(x-y) f(y) d y, \quad x>0,
$$

\footnotetext{
*Present Address, Hughes Aircraft Co., Culver City, Calif.

1 The preparation of this paper was sponsored (in part) by the Office of Naval Research. The content of this paper was submitted as partial fulfillment of the requirements for the degree of doctor of philosophy at the University of California at Los Angeles.

2 Figures in brackets indicate the literature references at the end of this paper.
} 
where $f(x)$ is unknown while $K(x)$ and $g(x)$ are known functions. In the electromagnetic problems $f(x)$ is the surface current density on the obstacle while, in the acoustic case, it represents the discontinuity in the field variable on opposite sides of the obstacle. Generally $g(x)$ is determined by the boundary conditions and the incident fields; it is zero in the present acoustic problem. The components of the field vectors at all points in space can be readily related to the Fourier transform of $f(x)$, which is obtained when the above equation is solved by the transform techniques of the Wiener-Hopf method.

Schwinger [1] also points out that if incident dominant mode waves fall upon an obstacle, the effect of the obstacle is to generate a far field of scattered or reflected lowest mode waves and a local field that is a result of the excitation of the attenuated higher modes. Although a complete description would include both fields in detail, as a practical guide problem, only the far field effect on the propagating modes need be considered. Another satisfactory method of representing this effect is by the use of equivalent circuits, by means of which a discontinuity in a wave guide is replaced by a lumped parameter network in a set of transmission lines.

It will be the purpose of this paper to apply the above methods to an infinite circular guide with a concentric semi-infinite circular bifurcating cylinder and to study the effect of such a discontinuity upon plane longitudinal waves by replacing it by an equivalent circuit. By a consideration of the equations that govern them, the unknown parameter in this network will be shown to reduce to one single essential parameter. This parameter will then be related to a distance $d$, which is the length the inner guide must be extended to account for the disturbance occurring in the immediate vicinity of the discontinuity caused by the excitation of higher mode fields. By this extension, we are able to introduce a new reference plane, the "virtual plane of bifurcation." Here the one essential parameter vanishes, and the equivalent circuit reduces to three transmission lines in series as shown in figure 6 . Thus all measurements made at a distance will indicate that the bifurcation occurs in the virtual plane that is $d$ units from the mouth of the inner guide, provided we assume that the discontinuity produces no disturbance.

The solution to the problem will be obtained by solving Helmboltz's equation subject to boundary conditions on the acoustic velocity potential at rigid walls. This is to be done with the aid of Green's theorem and the Green's function for a point source in the large guide. From the integrals in Green's theorem, we obtain an integral representation of the desired velocity potential. This integral we shall solve in its Fourier integral form with the aid of the homogeneous integral equation that is obtained when boundary conditions on the inner guide are applied to the integral representation. The homogeneous equation is of the above-mentioned Wiener-Hopf type; since $g(x)=0$, a solution by Fourier transforms is possible and yields an explicit expression for the transform of the unknown function. As the transform of the unknown appears in the Fourier integral form of the integral representation, the solution of the Wiener-Hopf equation permits a rigorous result for the velocity potential anywhere in all three regions (large guide, small guide, and coaxial guide).

It is also noteworthy that an alternative integral equation formulation [3] of the problem can be given. This originates with the division of the space interior to the large guide into the two regions $r>a, r<a$, where $a$ is the radius of the smaller guide. Green's theorem is then applied to each region with its appropriate Green's function, and each velocity potential is then expressed for $z<0$ in terms of its radial derivative on the surface $r=a$; here $z$ is a distance along the guide with $z=0$ at the mouth of the inner guide. The requirement of continuity for the velocity potential on crossing this surface provides an inhomogeneous integral equation of the Wiener-Hopf type for the determination of the common radial derivative.

Thus if we place the inner guide at $z \geq 0$, then, for $z<0$ and large, the integral representation reduces to linear expressions involving transmission line parameters and, for $z>0$ and large, it is given in terms of expressions from the Wiener-Hopf solution as well as a linear combination of transmission line parameters appropriate to the region considered. When the solutions to the right and left are then transformed back to the reference plane at the mouth of the inner guide, we find that the discontinuity can be represented as a six terminal network and that the 
one essential parameter and the distance $d$ can be also expressed in terms of these Wiener-Hopf results.

To simplify the problem to a considerable extent we are going to restrict the driving frequency to permit only one mode plane wave propagation in all three regions. In order to insure this type of propagation in the different regions, we must carefully examine the eigenvalues that arise in the solution of Helmholtz's equation in these regions. For the present problem where the normal derivative of the velocity potential vanishes at rigid walls, such a study will indicate that if we wish the propagating mode to be the lowest "axially symmetric" mode, we must have $0<k<x_{1} / b$ where $x_{1}$ is the smallest zero of the Bessel function $J_{1}(x)$. Since $\pi<x_{1}=3.832$, . . . , we may therefore select $\pi / b$ as a working upper limit to guarantee only principal-mode propagation in all regions.

\section{Description of Physical Quantities}

In studying the effect of the discontinuity upon a plane acoustic wave in an infinite circular guide, radius $b$, which contains a concentric semi-infinite coaxial guide of negligible thickness, radius $a$, we will assume that all the walls are perfectly rigid. Accordingly, the radial component of the particle velocity vanishes on the rigid walls. Thus, the scalar velocity potential $\Phi$ satisfies the wave equation

$$
\frac{\partial^{2} \Phi}{\partial t^{2}}=c^{2} \nabla^{2} \Phi
$$

where $c$ is the velocity of sound propagation in free space, and the partial derivatives of $\Phi$ with respect to the coordinates are the respective components of the velocity. A solution under the assumption of harmonic time dependence and complete angular symmetry about the z-axis can be given as

where

$$
\Phi(r, z, t)=\phi(r, z) e^{-i k c t}
$$

$$
k=\frac{2 \pi}{\lambda}
$$

and $\lambda=$ free space wavelength. Equation (1) then becomes the scalar Helmholtz equation

$$
\left(\nabla^{2}+k^{2}\right) \phi=\frac{\partial^{2} \phi}{\partial r^{2}}+\frac{1}{r} \frac{\partial \phi}{\partial r}+\frac{\partial^{2} \phi}{\partial z^{2}}+k^{2} \phi=0,
$$

subject to the boundary condition

$$
\frac{\partial \phi}{\partial r}=0 \text { at }\left\{\begin{array}{l}
r=b \text { for all } z \\
r=a \text { for } z>0 .
\end{array}\right.
$$

Once $\phi$ is known, we can calculate the other important acoustic quantities from the classic relationships between the velocity potential, pressure, density of gas, and condensation.

We now formulate the equation which expresses the field variable in terms of the discontinuity of $\phi$ across the surface of the inner guide (surface current density in electromagnetic case). To do this, we are going to make use of Green's theorem to construct the Green's function for the structure at hand out of a more readily obtainable one, which in our problem is the one for the empty guide of radius $b$. Thus we are considering the inner guide as an obstacle embedded in the space of the other. The appropriate Green's function which will aid appreciably in the solution of the differential equation in $\phi$ satisfies a similar but inhomogeneous equation with the simpler boundary condition

$$
\frac{\partial G}{\partial r}=0 \text { at } r=b \text { for all } z \text {. }
$$


Therefore, Green's function for the velocity potential, with no angular dependence, satisfies the equation

$$
\left(\nabla^{2}+k^{2}\right) G\left(r, r^{\prime}, z-z^{\prime}\right)=-\frac{\delta\left(r-r^{\prime}\right)}{r} \delta\left(z-z^{\prime}\right)
$$

Here $\delta\left(x-x^{\prime}\right)$ is the Dirac delta function and is zero everywhere save at $x-x^{\prime}$, where it becomes infinite in such a fashion as to make

$$
\int_{-\infty}^{\infty} \delta\left(x-x^{\prime}\right) d x^{\prime}=1
$$

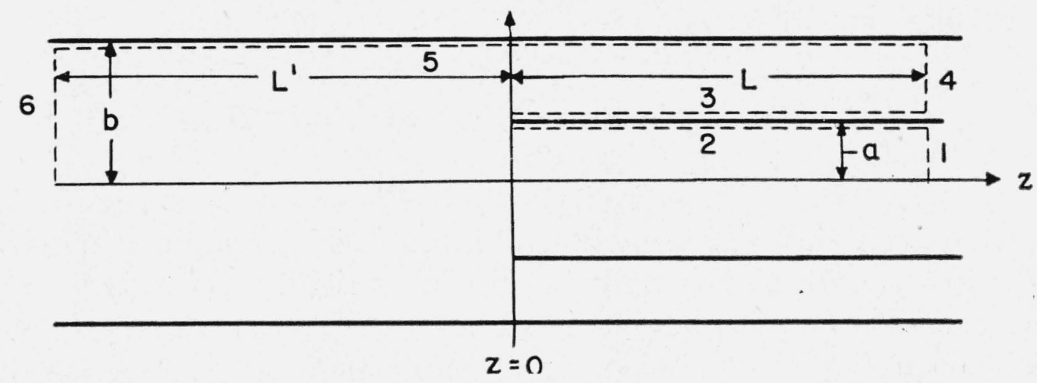

Figure 2.

In applying Green's theorem, we take the region under consideration as that which is enclosed by the dotted line in figure 2 and write

$$
\int_{V^{\prime}}\left[G \left(\nabla^{\prime 2}+k^{2} \phi-\phi\left(\nabla^{\prime 2}+k^{2} G\right] d V^{\prime}=\int_{S^{\prime}}\left(G \frac{\partial \phi}{\partial n^{\prime}}-\phi \frac{\partial G}{\partial n^{\prime}}\right) d S^{\prime}\right.\right.
$$

where $n^{\prime}=$ outward normal to the bounding surface. Although $d V^{\prime}$ represents an element of volume enclosed by the dotted line and $d S^{\prime}$ an element of surface area, the problem can be reduced to two dimensions by immediately integrating with respect to the angle, since there is complete circular symmetry. This integration merely brings in a factor of $2 \pi$ on both sides of the above and removes the angle dependence from all subsequent equations. $G$ here is an appropriate Green's function that satisfies (6) and (5).

To evaluate (8), we impose boundary conditions (4) and (5) together with (3) and (6). Since the volume integral reduces to $\phi(r, z)$, Green's identity yields

$$
\phi(r, z)=\int_{S^{\prime}}\left(G \frac{\partial \phi}{\partial n^{\prime}}-\phi \frac{\partial G}{\partial n^{\prime}}\right) d S^{\prime}
$$

where $S^{\prime}$ is determined by the six regions in figure 2 and $n^{\prime}$ measures distance along the outward normal to the surface enclosed by dotted line. By writing $G^{-}\left(r, r^{\prime}, z-z^{\prime}\right)$ as theasymptotic form of Green's function for $\mathrm{z}^{\prime}$ large and negative and $G^{+}\left(r, r^{\prime}, z-z^{\prime}\right)$ as the asymptotic form for $z^{\prime}$ large and positive, (9) becomes the sum of the six integrals,

$$
\begin{aligned}
& \int_{1}=\int_{0}^{a}\left[G^{+} \frac{\partial \phi}{\partial z^{\prime}}-\phi \frac{\partial G^{+}}{\partial z^{\prime}}\right]_{z^{\prime}=L} r^{\prime} d r^{\prime} \\
& \int_{2}=\int_{0}^{L}\left[-\phi\left(a-0, z^{\prime}\right) \frac{\partial G}{\partial r^{\prime}}\right]_{r^{\prime}=a} a d z^{\prime} \\
& \int_{3}=\int_{0^{\circ}}^{L}-\left[-\phi\left(a+0, z^{\prime}\right) \frac{\partial G}{\partial r^{\prime}}\right]_{r^{\prime}=a} a d z^{\prime},
\end{aligned}
$$


(minus in front since $n^{\prime}=-r^{\prime}$ ),

$$
\begin{aligned}
& \int_{4}=\int_{a}^{b}\left[G^{+} \frac{\partial \phi}{\partial z^{\prime}}-\phi \frac{\partial G^{+}}{\partial z^{\prime}}\right]_{z^{\prime}=L} r^{\prime} d r^{\prime} \\
& \int_{5}=\int_{-L^{\prime}}^{L}\left[G \frac{\partial \phi}{\partial r^{\prime}}-\phi \frac{\partial G}{\partial r^{\prime}}\right]_{r^{\prime}=b} b d z^{\prime}=0 \\
& \int_{6}=\int_{0}^{b}-\left[G-\frac{\partial \phi}{\partial z^{\prime}}-\phi \frac{\partial G^{-}}{\partial z^{\prime}}\right]_{z^{\prime}=-L^{\prime}} r^{\prime} d r^{\prime}
\end{aligned}
$$

(minus in front since $n^{\prime}=-z^{\prime}$ ).

Since it will be shown later that all integrals taken along transverse surfaces are independent of $L$ or $L^{\prime}$ as the case may be, we may now let $L$ and $L^{\prime} \rightarrow \infty$ and write (10) as

$$
\begin{gathered}
\phi(r, z)=\int_{0}^{\infty}\left(\phi^{+}-\phi^{-}\right)\left(\frac{\partial G}{\partial r^{\prime}}\right)_{r^{\prime}=a} a d z^{\prime}+\lim _{L \rightarrow \infty}\left[\int_{0}^{a}\left(G^{+} \frac{\partial \phi}{\partial z^{\prime}}-\phi \frac{\partial G^{+}}{\partial z^{\prime}}\right)_{z^{\prime}=L}\right. \\
\left.+\int_{a}^{b}\left(G^{+} \frac{\partial \phi}{\partial z^{\prime}}-\phi \frac{\partial G^{+}}{\partial z^{\prime}}\right)_{z^{\prime}=L} r^{\prime} d r^{\prime}-\int_{0}^{b}\left(G^{-} \frac{\partial \phi}{\partial z^{\prime}-\phi} \frac{\partial G^{-}}{\partial z^{\prime}}\right)_{z^{\prime}=-L^{\prime}} r^{\prime} d r^{\prime}\right]
\end{gathered}
$$

The difference of the velocity potential on the inner and outer surfaces of the small guide which appears in the first integral of (11) is a consequence of the oppositely directed normals at these surfaces.

\section{Derivation of the Appropriate Green's Function}

To obtain the solution $G\left(r, r^{\prime}, z\right)$ of $(6)$ in the total space interior to the larger guide, we shall apply standard Fourier transform techniques. Thus, we transform $z$ out of (6) by defining the Fourier transform in $z$ as

$$
\mathscr{G}\left(r, r^{\prime}, \zeta\right)=\int_{-\infty}^{\infty} e^{-i \zeta z} G\left(r, r^{\prime}, z\right) d z,
$$

where we have written $z$ instead of $z-z^{\prime}$, since this binomial appears explicitly in the solution, $\mathscr{G}$ now satisfies the equation

$$
\left(\frac{\partial^{2}}{\partial r^{2}}+\frac{1}{r} \frac{\partial}{\partial r}+\gamma^{2}\right) \mathscr{G}\left(r, r^{\prime}, \zeta\right)=\frac{-\delta\left(r-r^{\prime}\right)}{r},
$$

where $\gamma^{2}=k^{2}-\zeta^{2} ; \mathscr{G}$ is subject to the boundary conditions: (1) that it be finite at the origin and (2) that its normal derivative vanish at $r=b$. Thus, we must seek a solution of Bessel's equation of order zero, since there is angular symmetry, which satisfies these boundary conditions and is continuous at $r=r^{\prime}$. Following Courant-Hilbert [4], the solution of (13) can be given as

$$
\left.\begin{array}{ll}
\mathscr{G}\left(r, r^{\prime}, \zeta\right)=\frac{\pi}{2} \frac{J_{0}\left(\gamma r^{\prime}\right)}{J_{1}(\gamma b)}\left[J_{0}(\gamma r) N_{1}(\gamma b)-N_{0}(\gamma r) J_{1}(\gamma b)\right] & \left(r>r^{\prime}\right) \\
\mathscr{G}\left(r, r^{\prime}, \zeta\right)=\frac{\pi}{2} \frac{J_{0}(\gamma r)}{J_{1}(\gamma b)}\left[J_{0}\left(\gamma r^{\prime}\right) N_{1}(\gamma b)-N_{0}\left(\gamma r^{\prime}\right) J_{1}(\gamma b)\right] . & \left(r<r^{\prime}\right)
\end{array}\right\}
$$

By the Mellin inversion, these then yield $G$ in the form

$$
G=\frac{1}{4} \int_{i \sigma-\infty}^{i \sigma+\infty} e^{+i \zeta z} d \zeta \frac{J_{0}(\gamma r<)}{J_{1}(\gamma b)}\left[J_{0}\left(\lambda r_{>}\right) N_{1}(\gamma b)-N_{0}(\gamma r>) J_{1}(\gamma b)\right]
$$


where the integration contour is a straight line in the region of regularity of the Green's function transform and $r_{>}, r_{<}$symbolizes the larger and smaller of the coordinates $r, r^{\prime}$, respectively.

To determine the region where the integral in (15) converges, we must first determine the form of $G$ and then apply it to (12) which defines $\mathscr{G}(\zeta)$. From our fundamental assumption of only one mode propagation, we know that

$$
G \sim A e^{i k|z|}+\sum_{n=1}^{\infty} B_{n} e^{-K_{n}|z|} .
$$

It can be easily shown that for a $G$ of this form, the integral in (12) has no region of regularity unless $k$, the propagation constant, has an arbitrarily small positive imaginary part (which is eventually set equal to zero). This corresponds to a small attenuation of the sound waves traveling in the guide. Thus, if we let $k=k_{1}+i \epsilon$ and introduce the coordinates in the $\zeta$-plane as $\zeta=\xi+i \eta$, (12) converges in the strip $|\eta|<\epsilon(\operatorname{Im} k)$. Consequently, the inversion given in (15) can be performed, if $\sigma$ is restricted to this same strip.

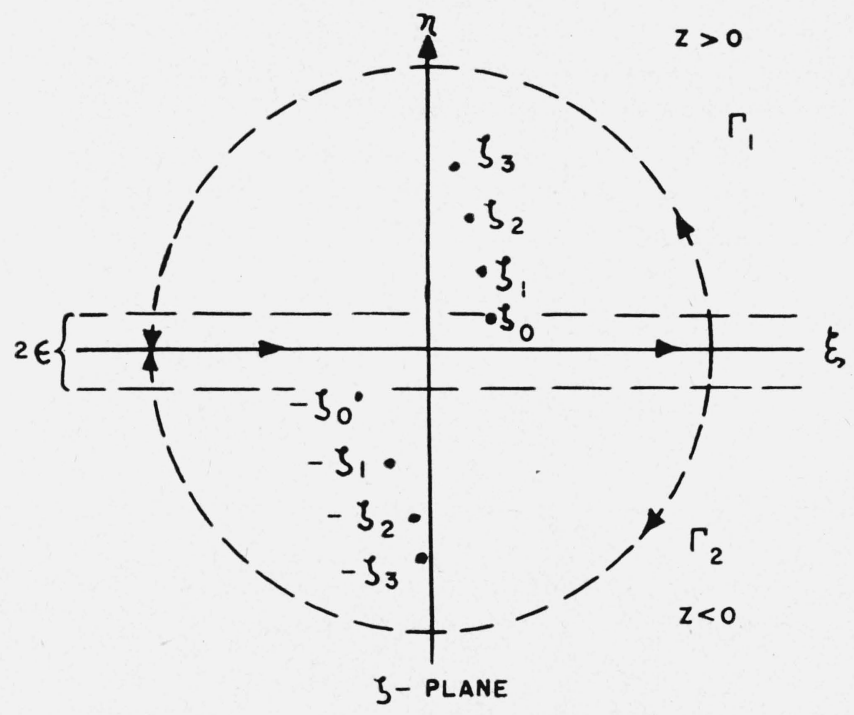

Figure 3.

The integral in (15) may now be evaluated by computing the residues inside the semicircular contours pictured in figure 3. The poles of (15) which are given by the zeros of $J_{1}(\gamma b)$ are all complex for $\epsilon>0$. Thus, if we let $J_{1}\left(\gamma_{n} b\right)=J_{1}\left(x_{n}\right)=0$ for $n=0,1,2,3, \ldots$ and $\zeta_{n}=$ $k^{2}-\gamma_{n}^{2}$, compute the residues inside $\Gamma_{1}$ and $\Gamma_{2}$, and then let $\epsilon \rightarrow 0$, we find that the solution of (6) becomes

$$
G=\frac{i e^{i k\left|z-z^{\prime}\right|}}{k b^{2}}+\sum_{n=1}^{\infty} \frac{J_{0}\left(x_{n} \frac{r}{b}\right) J_{0}\left(x_{n} \frac{r^{\prime}}{b}\right) e^{-K_{n}\left|z-z^{\prime}\right|}}{\kappa_{n} b^{2} J_{0}^{2}\left(x_{n}\right)} .
$$

Here we have insured only one mode propagation by restricting $k$ to be less than $x_{1} / b$ and defining the attenuation constants by $\mathbf{K}_{n}^{2}=\left(x_{n} / b\right)^{2}-k^{2}$ where $\mathrm{K}_{1}^{2}=\left(x_{1} / b\right)^{2}-k^{2}$ is the smallest.

The Green's function which is appropriate to this problem must give a physical description of waves incident upon and reflected from a discontinuity in a guide. Since (17) represents a wave traveling outward from a source, the appropriate Green's function requires a superposition of (17) and its complex conjugate which is the solution of (6) representing a wave traveling inward to a sink. Thus if we take one half of the sum of (17) and its conjugate and simplify the results, we obtain

$$
G\left(r, r^{\prime}, z-z^{\prime}\right)=-\frac{\sin k\left|z-z^{\prime}\right|}{k b^{2}}+\sum_{n=1}^{\infty} \frac{J_{0}\left(x_{n} \frac{r}{b}\right) J_{0}\left(x_{n} \frac{r^{\prime}}{b}\right) e^{-K_{n}\left|z-z^{\prime}\right|}}{\mathbf{K}_{n} b^{2} J_{0}^{2}\left(x_{n}\right)},
$$

which has the desired physical properties. 


\section{Evaluation of Integrals}

Once the appropriate Green's function has been determined, we can proceed to evaluate the integrals in (11). This is done in paragraphs (a) and (b), since the character of the results and the evaluation techniques used differ widely. Paragraph (a) is concerned with obtaining the far field contribution in terms of the transmission line parameters and paragraph (b) with the setting up of the Wiener-Hopf integral equation which is a result of the first integral in (11).

(a) In proceeding, let us temporarily ignore the first integral of (11) and examine the last three. From the explicit form of the Green's function, (18), the asymptotic forms of $G$ can be given as

$$
G^{+}\left(r, r^{\prime}, z-z^{\prime}\right)=G^{-}\left(r^{\prime}, r, z-z^{\prime}\right)=-\frac{\sin k\left|z-z^{\prime}\right|}{k b^{2}} .
$$

Thus, when we examine (11) for $L$ and $L^{\prime}$ sufficiently large so that the attenuated terms may be considered negligible, only the forms in (19) need be used. It can be shown [5] that in terms of the propagation of acoustic waves in transmission tubes, the velocity potential and pressure obey analogous equations to those for currents and voltages in a transmission line. Thus, for $z$ large, $\phi$ behaves as the current in a transmission line which we will denote as $I(z)$. Then, in terms of the transmission line currents and voltages measured at $z=0$, we write

$$
\phi(r, z) \sim I(z)=[I \cos k z-i V Y \sin k z],
$$

where

$$
Y=\frac{1}{z}=\frac{1}{\rho c}
$$

is the characteristic admittance of the line and $\rho$ is the density of the air or the gas in the tubes.

Using (20) for each of the three regions under consideration, we note that as $z \rightarrow-\infty$

$$
\phi(r, z) \sim I_{1}(z)=\left[I_{1} \cos k z+i V_{1} Y_{1} \sin k z\right],
$$

while for $z \rightarrow \infty$ and for $r<a$

$$
\phi(r, z) \sim I_{3}(z)=\left[I_{3} \cos k z-i V_{3} Y_{3} \sin k z\right],
$$

and for $z \rightarrow \infty$ and for $b>r>a$

$$
\phi(r, z) \sim I_{2}(z)=\left[I_{2} \cos k z-i V_{2} Y_{2} \sin k z\right] .
$$

Thus, the last integral of (11) becomes

since

$$
\lim _{L^{\prime} \rightarrow \infty}\left[-\int_{0}^{b}\left(G^{-} \frac{\partial \phi}{\partial z^{\prime}}\left(r^{\prime}, z^{\prime}\right)-\phi \frac{\partial G^{-}}{\partial z}\right)_{z^{\prime}=-L^{\prime}} r^{\prime} d r^{\prime}\right]=\frac{I_{1}(z)}{2}
$$

$$
\frac{\partial \phi}{\partial z^{\prime}}=k\left(-I_{1} \sin k z^{\prime}+i V_{1} Y_{1} \cos k z^{\prime}\right), \quad G^{-}=-\frac{\sin k\left(z-z^{\prime}\right)}{k b^{2}}, \quad \frac{\partial G^{-}}{\partial z^{\prime}}=\frac{1}{b^{2}} \cos k\left(z-z^{\prime}\right) .
$$

Likewise,

$$
\lim _{L \rightarrow \infty}\left[\int_{0}^{a}\left(G^{+} \frac{\partial \phi}{\partial z^{\prime}}-\phi \frac{\partial G^{+}}{\partial z^{\prime}}\right)_{z^{\prime}=L} r^{\prime} d r^{\prime}\right]=\left(\frac{a^{2}}{b^{2}}\right) \frac{I_{3}(z)}{2}
$$

and

since

$$
\lim _{L \rightarrow \infty}\left[\int_{a}^{b}\left(G^{+} \frac{\partial \phi}{\partial z^{\prime}}-\phi \frac{\partial G^{+}}{\partial z^{\prime}}\right)_{z^{\prime}=L} r^{\prime} d r^{\prime}\right]=\left(\frac{b^{2}-a^{2}}{b^{2}}\right) \frac{I_{2}(3)}{2}
$$

$$
\frac{\partial \phi}{\partial z^{\prime}}=k\left(-I_{3} \sin k z-i V_{3} Y_{3} \cos k z^{\prime}\right), \quad G^{+}=-\frac{\sin k\left(z^{\prime}-z\right)}{k b^{2}}, \quad \frac{\partial G^{+}}{\partial z^{\prime}}=-\frac{\cos k\left(z^{\prime}-z\right)}{b^{2}} .
$$


Thus (11) becomes

$$
\phi(r, z)=\int_{0}^{\infty} H\left(z^{\prime}\right)\left(\frac{\partial G}{\partial r^{\prime}}\right)_{r^{\prime}=a} d z^{\prime}+\frac{1}{2}\left[I_{1}(z)+\left(\frac{b^{2}-a^{2}}{b^{2}}\right) I_{2}(z)+\left(\frac{a^{2}}{b^{2}}\right) I_{3}(z)\right]
$$

where

$$
H(z)=a\left[\phi^{+}(r, z)_{r=a+0}-\phi^{-}(r, z)_{r=a-0}\right] .
$$

Since (28) is independent of $L$ and $L^{\prime}$ we, therefore, note that all the integrals taken along transverse surfaces yield propagating terms which depend only on z. Equation (28) represents the velocity potential at all points in the large guide in terms of the potentials of the three far fields and the potential of the unknown scattered field which is given by the integral.

(b) Since $H(z)$ is as yet an unknown function, (27), the equation we referred to in our introduction as the integral representation of the desired velocity potential, is an inhomogeneous integral equation. When we impose the acoustic boundary condition

$$
\left[\frac{\partial \phi}{\partial r}\right]_{r=a}=0, \quad \text { for } z \geq 0,
$$

this equation becomes readily soluble by transform methods since it is homogeneous and of the convolution type.

Thus, if we call

$$
V(z)=\left(\frac{\partial \phi}{\partial r}\right)_{r=a} \quad \text { for } z<0
$$

and since the propagating terms drop out in the differentiation, we have from (27)

$$
\begin{aligned}
& \left.0=\int_{-\infty}^{\infty} K\left(z-z^{\prime}\right) H\left(z^{\prime}\right) d z^{\prime}, \quad z \geq 0\right\} \\
& \left.V(z)=\int_{-\infty}^{\infty} K\left(z-z^{\prime}\right) H\left(z^{\prime}\right) d z^{\prime}, \quad z<0\right\}
\end{aligned}
$$

where the range of integration has been extended to $-\infty$, since, from (28)

$$
H(z)= \begin{cases}0, & z<0 \\ H(z), & z \geq 0 .\end{cases}
$$

Equation (29) is a homogeneous integral equation that resembles Wiener-Hopf type, since the dependence of $G$ and therefore the kernel,

$$
K\left(z-z^{\prime}\right)=\left[\frac{\partial}{\partial r}\left\{\frac{\partial G}{\partial r^{\prime}}\left(r^{\prime}, r, z-z^{\prime}\right)\right\}\right]_{r=r^{\prime}=a},
$$

upon $z$ is of the form $\left(z-z^{\prime}\right)$.

\section{Derivation of the Lumped Parameter Circuit and the One Essential Parameter}

From equation $(27)$, we can now derive the circuit relation of a lumped parameter network with which the discontinuity can be replaced, if we concern ourselves with fields far enough away from the mouth of the inner guide so that all attenuated modes have essentially vanished. To investigate this far field result on the left as $z \rightarrow-\infty$, we let $\phi(r, z)$ be given by the transmission line expression (21) and define the characteristic admittances as inversely proportional to the area:

$$
\frac{1}{Z_{1}}=Y_{1} \sim \frac{1}{\pi b^{2}} ; \quad \frac{1}{Z^{2}}=Y_{2} \sim \frac{1}{\pi\left(b^{2}-a^{2}\right)} ; \quad \frac{1}{Z^{3}}=Y_{3} \sim \frac{1}{\pi a^{2}} .
$$


Thus, as we shall see later, the integral in (27) vanishes for $z<0$ and large and we are left with

which becomes

$$
I_{1}(z)=\frac{1}{2}\left[I_{1}(z)+I_{2}(z) \frac{Z_{2}}{Z_{1}}+I_{3}(z) \frac{Z_{3}}{Z_{1}}\right]
$$

$$
Z_{2} I_{2}(z)+Z_{3} I_{3}(z)-Z_{1} I_{1}(z)=0
$$

Now, if we rewrite the above in terms of the $I$ 's and $V$ 's at $z=0$, we have

$Z_{2}\left(I_{2} \cos k z-i V_{2} Y_{2} \sin k z\right)+Z_{3}\left(I_{3} \cos k z-i V_{3} Y_{3} \sin k z\right)-Z_{1}\left(I_{1} \cos k z+i V_{1} Y_{1} \sin k z\right)=0$,

and since the sine and cosine are linearly independent functions, (31) becomes

and

$$
Z_{2} I_{2}+Z_{3} I_{3}-Z_{1} I_{1}=0
$$

These two, together with

$$
V_{1}+V_{3}+V_{3}=0 \text {. }
$$

$$
Z_{1}=Z_{2}+Z_{3}
$$

a result of (30), are the defining equations for a six terminal lumped parameter circuit. Thus we have three relationships between the circuit parameters which were deduced by examining the effect on the velocity potential as $z \rightarrow-\infty$ and which represent the far field results to the left. We will presently determine the far field results to the right and then by expressing them in terms of the current and voltages at the zero reference plane, we will find that the extrapolated results coming from the left and right can be represented by same six terminal impedance network.

In general, a six terminal network contains nine impedance parameters representing the ratios of voltages to currents for all possible combinations of the three independent voltages and currents. However, if we make use of the reciprocity theorem (see Schwinger [1] or Everett [6]) of electrical circuit theory the number of impedance parameters is reduced to six. From the above three equations, we further reduce this number to three, which we will call the susceptances, $B_{1}, B_{2}$, and $B_{3}$. Also, from (34), we notice that they must be proportional to $Y_{1}, Y_{2}$, and $Y_{3}$, respectively. If this circuit is to satisfy the above equations, it must have the following delta form (fig. 4) with nondissipative pure reactive elements and with the power flow arbitrarily chosen to flow into the junction from both sides.

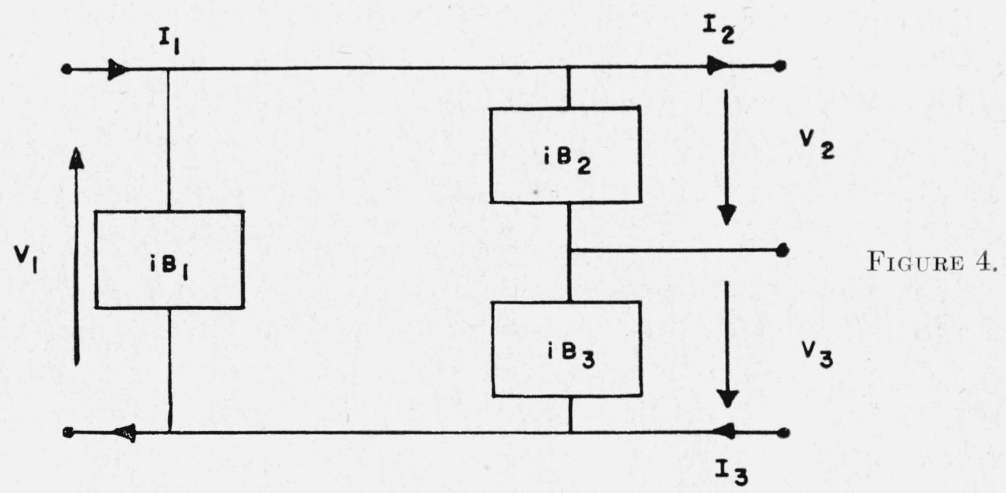

Since the circuit parameters are independent of the impressed currents and voltages, we can now obtain independent relationships between the $B$ 's as follows:

1. Open circuit lines 2 and 3. Thus $I_{2}=I_{3}=0$ and from (32), $I_{1}=0$. By looking into line 1 , we see $B_{1}$ in parallel with the series combination of $B_{2}$ and $B_{3}$ and we have

$$
\left\{i B_{1}+\frac{1}{\frac{1}{i B_{2}}+\frac{1}{i B_{3}}}\right\}=0 \text { or }\left[\frac{1}{B_{1}}+\frac{1}{B_{2}}+\frac{1}{B_{3}}\right]=0 \text {. }
$$


2. If we terminate lines 2 and 3 by their characteristic impedance, it can be easily shown that the impedance seen from line 1 is the characteristic impedance

$$
Y_{1}=i B_{1}+\frac{\left(i B_{2}+Y_{2}\right)\left(i B_{3}+Y_{3}\right)}{\left(i B_{2}+i B_{3}+Y_{2}+Y_{3}\right)} .
$$

With the independent derivation of these last two equations, we see that the number of independent parameters is now one. Thus from the proportionality required by (34), we let

Then from (36), we obtain

$$
B_{1}=b_{1} Y_{1}, B_{2}=b_{2} Y_{2} \text {, and } B_{3}=b_{3} Y_{3} \text {. }
$$

$$
\frac{1}{Y_{1}\left(1-i b_{1}\right)}=\frac{1}{Y_{2}\left(i b_{2}+1\right)}+\frac{1}{Y_{3}\left(i b_{3}+1\right)}
$$

and (35) becomes

$$
\left[\frac{1}{Y_{1} b_{1}}+\frac{1}{Y_{2} b_{2}}+\frac{1}{Y_{3} b_{3}}\right]=0
$$

By solving (37), (38), and (34) simultaneously for the three unknown parameters, we find that

and figure 4 reduces to figure 5 .

$$
b_{1}=-b_{2}=-b_{3}=B
$$

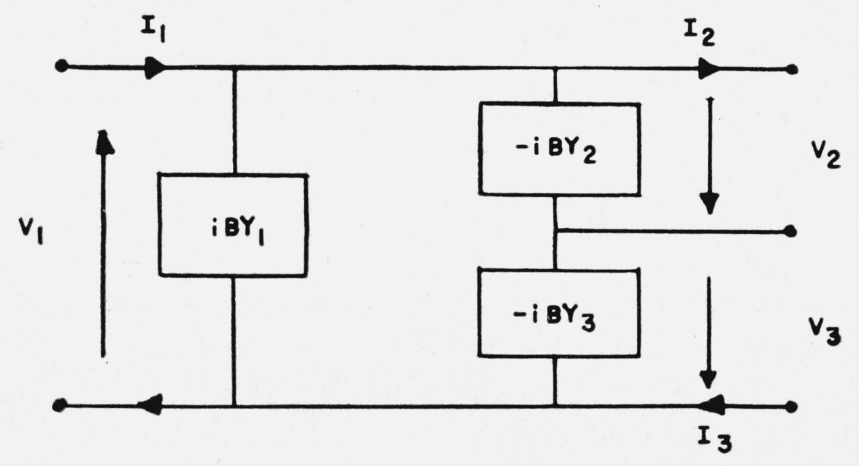

Figure 5.

Thus we have shown that with respect to the far field results on the left, the discontinuity can be replaced by the above network. To determine the effect on the right as $z \rightarrow+\infty$, we must first evaluate the integral in (27). A straight forward circuit analysis of figure 5 shows that $B$ is related to the circuit parameters by

$$
B=\frac{i\left(I_{2}-I_{1}\right)}{V_{1} Y_{1}+V_{2} Y_{2}} .
$$

We have now shown that the circuit in figure 5 can be used to represent the discontinuity, when the parameters are measured from the $z=0$ reference plane which is at the mouth of the inner guide. Since, there is only one essential circuit parameter which has a known variation with driving frequency and the distance to the reference, it is possible to find a reference plane in which the equivalent circuit susceptances vanish and, consequently, the equivalent circuit reduces to three transmission lines in series as shown in figure 6 .

This new reference plane is a "virtual plane of bifurcation", inasmuch as all measurements made at a distance will indicate that the bifurcation occurs in this plane, provided we neglect the effect of the discontinuity. Particularly, if one of the tubes is introduced in order to take measurements, the quantities will be measured in the virtual plane and not at the mouth of the tube.

To find this virtual plane which is at $z=-d$, or a distance $d$ "ahead" of the actual plane of bifurcation, we follow the method outlined by Miles [7] and let $\eta==k d$ and transform the 


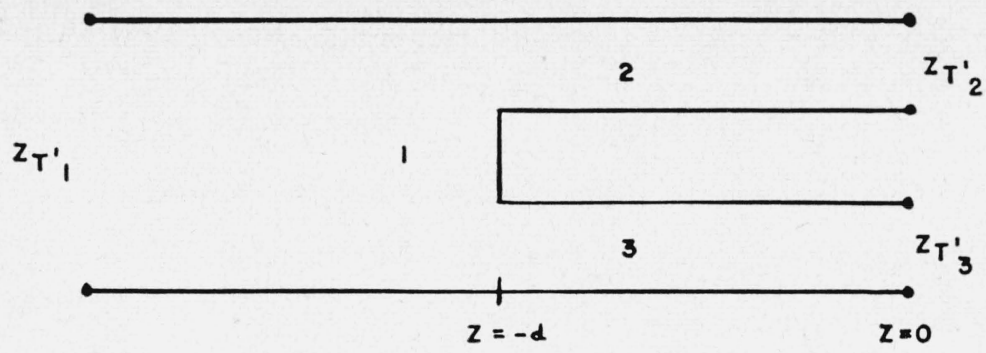

Figure 6.

impedance seen by the $i$-th tube $(i=1,2,3),, Z_{T_{i}}$ (plane $\left.z=0\right)$, to $Z_{T_{i}}^{\prime}$ (plane $z=-d$ ). This transformation is effected by the use of

$$
\frac{Z_{T_{i}}^{\prime}}{Z_{i}}=\frac{ \pm \frac{Z_{T_{i}}}{Z_{i}} \cos \eta+i \sin \eta}{ \pm \cos \eta+i\left(\frac{Z_{T_{i}}}{Z_{i}}\right) \sin \eta}
$$

but care must be taken to select the proper signs (both plus if the direction of the power flow obtained by following $V_{i}$ then $I_{i}$ is in the positive $z$ direction) and to use a negative $\eta$ since the reference plane is being moved to $z=-d$. Thus, if we load terminals 2 and 3 (fig. 5 ) with arbitrary admittances $Y_{T_{2}}$ and $Y_{T_{3}}$, it can be easily shown that line 1 sees

$$
Y_{T_{1}}=\frac{1}{Z_{T_{1}}}=i B Y_{1}+\frac{\left[Y_{T_{2}}-i B Y_{2}\right]\left[Y_{T_{3}}-i B Y_{3}\right]}{\left[Y_{T_{3}}+Y_{T_{2}}-i B\left(Y_{3}+Y_{2}\right)\right]} .
$$

Thus if

$$
Z_{T_{3}}=0=\frac{1}{Y_{T_{3}}} \quad \text { and } \quad Y_{T_{2}}=0
$$

then from $(42)$

$$
Z_{T_{1}}=\frac{1}{i B\left(Y_{1}-Y_{2}\right)}
$$

Now, if we transform the above $Z_{T_{i}}$ to $Z_{T_{i}}^{\prime}$ until the circuit can be given as the series combination (fig. 6), namely,

$$
Z_{T_{1}}^{\prime}=Z_{T_{2}}^{\prime}+Z_{T_{3}}^{\prime}
$$

we find that

$$
B=\tan \eta
$$

\section{Fourier Transform Solution of the Integral Equation}

The Fourier transform solution of (29) will be undertaken as follows: In part (a) of the present section, the equation linking the transforms of the respective functions $V, H$, and $K$ and the common region of regularity of these transforms will be derived. The equation follows as a direct consequence of the convolution theorem. 'The regions of analyticity of the transforms are obtained from the asymptotic forms of the functions themselves. Part (b) will concern itself with the decomposition of the transform of the kernel, $\mathrm{K}$, into the quotient of two functions which will convert the equation of the transforms into a single integral function. This integral function will, in part (c), be shown to be constant since it satisfies the conditions of the Liouville theorem. Thus from (70), we will be able to find an expression for the transform of the heretofore unknown function $H(z)$, and thereby, in part (d), we will obtain $\phi(r, z)$ from the inversion integral. 
(a) The solution of the extended Wiener-Hopf integral equation (29) by Fourier transform methods can be facilitated by the theorem of convolution which permits us to write the transform of the product of two functions as the product of the transforms. Hence, the transform of (29) becomes

where

$$
\mathscr{V}(\zeta)=\mathscr{K}(\zeta) \mathscr{H}(\zeta)
$$

$$
\mathscr{K}(\zeta)=\int_{-\infty}^{\infty} e^{-i \zeta\left(z-z^{\prime}\right)}\left[\frac{\partial}{\partial r}\left(\frac{\partial G}{\partial r^{\prime}}\right)\right]_{r=r^{\prime}=a} d\left(z-z^{\prime}\right)=\left[\frac{\partial}{\partial r}\left(\frac{\partial}{\partial r^{\prime}} \mathscr{G}\right)\right]_{r=r^{\prime}=a}
$$

and $\mathscr{H}(\zeta)$ and $\mathscr{V}(\zeta)$ denote the Fourier transforms of the respective functions, $H(z)$ and $V(z)$ in the form $f(\zeta)=\int_{-\infty}^{\infty} f(x) e^{-i \xi x} d x$. For the transform equation (47) to have significance, there must be a common domain of analyticity of the transforms in the $\zeta$-plane. To verify the existence and location of this domain, we examine each transform with the aid of the growth order of their corresponding functions $V(z), H(z)$ and $K(z)$.

Previously, we showed that $\mathscr{G}(\zeta)$ exists only if $k$ has an imaginary part. However, $\mathscr{K}(\zeta)$ involves differentiation with respect to $r$ and $r^{\prime}$ which eliminates the propagating term and, consequently its region of regularity is $|\eta|<\mathrm{K}_{1}$.

The quantity which we have called $H(z)$, given explicitly by

$$
\left[\phi(r, z)_{r=a+0}-\phi(r, z)_{r=a-0}\right] a
$$

has the same asymptotic form, for $z \rightarrow \infty$, as the velocity potential on the inner and outer surfaces of the smaller guide and, therefore, can be given as $H(z) \sim A e^{i k z}+B e^{-i k z}$. From this, we infer that $\mathscr{H}(\zeta)$ has only simple poles on the real axis at $\zeta= \pm k$, and is regular in the lower half plane $\eta<0$, since

is bounded in this region.

$$
|\mathscr{H}(\zeta)| \leq \int_{0}^{\infty}|H(z)| e^{\eta z} d z
$$

If we study the defining equation for $V(z)$, (29), by inserting the explicit expression for the asymptotic behavior of the kernel, namely $K\left(z-z^{\prime}\right) \sim e^{-K_{1}\left|z-z^{\prime}\right|}$, we see that the real part of $V(z) \sim e^{K_{1} z}$ as $z \rightarrow-\infty$, since $H(z)$ is the entire function (traveling wave) given above.

Thus

and exists for $\eta>-\mathrm{K}_{1}$.

$$
\mathscr{V}(\zeta) \sim \int_{-\infty}^{0} e^{-i \zeta z+K_{1} z} d z=\int_{-\infty}^{0} e^{-i \xi z+\left(\eta+K_{1}\right) z} d z
$$

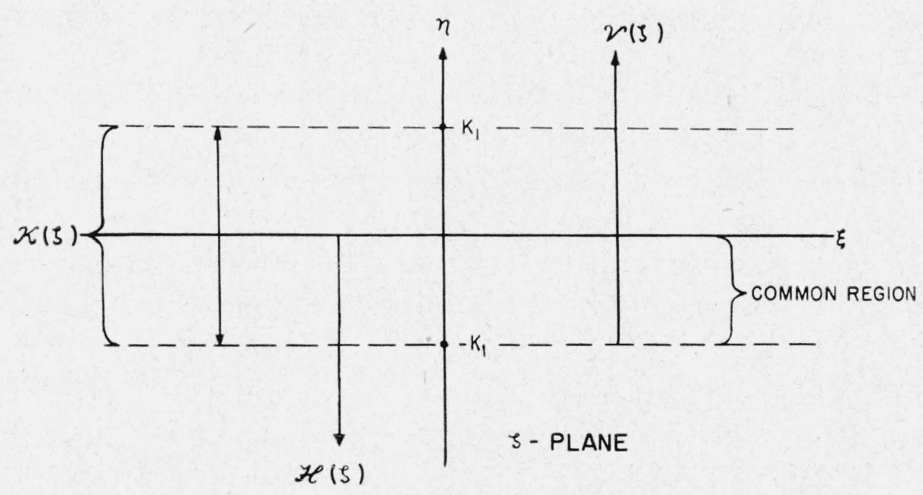

Figure 7. 
Collecting results, we find (fig. 7) that the strip $-\mathrm{K}_{1}<\eta<0$ is a common region of regularity for the transform equation (47). It will now be convenient to designate the region $\eta>-\mathrm{K}_{1}$ and $\eta<0$ as the upper and lower half planes respectively.

(b) In order to solve the transform equation (47), we now seek to represent $\mathscr{K}(\zeta)$ as a quotient of functions $K_{-}(\zeta)$ and $K_{+}(\zeta)$ such that a simple transposition will make each side of the equation regular and not zero in separate half planes with a common strip. By writing (47) as

$$
\mathscr{V}(\zeta) K_{+}=\mathscr{H}(\zeta) K_{-}=\text {integral function, }
$$

we will see that each side of the equation is the analytic continuation of the other and therefore will represent the same function. Together they will then define an analytic function throughout the finite $\zeta$-plane. If, in addition, this analytic integral function turns out to be bounded in the whole plane, then, as a consequence of the Liouville theorem [8], it must be equal to a constant and the transforms $\mathscr{H}(\zeta)$ and $\mathscr{V}(\zeta)$ are simply related to $K_{+}$and $K_{-}$.

Although there are general methods for the decomposition of the kernel which are both rigorous and forceful and have been successfully [3, 9] used, in our present discussion however, a simpler and more direct approach will suffice as it has in other problems [10]. Thus we consider

$$
\mathscr{K}(\zeta)=\left[\frac{\partial}{\partial r}\left\{\frac{\partial}{\partial r^{\prime}} \mathscr{G}(\zeta)\right\}\right]_{r=r^{\prime}=a}=\frac{\pi}{2} \gamma^{2}\left[\frac{J_{1}(\gamma a)}{J_{1}(\gamma b)}\right]\left[J_{1}(\gamma a) N_{1}(\gamma b)-J_{1}(\gamma b) N_{1}(\gamma a)\right]
$$

and write each factor as an infinite product of the form $\left(a^{2}-b^{2}\right)$ which we will simply split into terms which have simple zeros in one half plane or the other.

Thus to convert (52) to infinite products, we start by letting

$$
f(\gamma)=J_{1}(\gamma a) N_{1}(\gamma b)-J_{1}(\gamma b) N_{1}(\gamma a)
$$

From Whittaker and Watson [11], this may also be written as

$$
f(\gamma)=C \prod_{n=1}^{\infty}\left(1-\frac{\gamma^{2}}{\gamma_{n}^{2}}\right)
$$

where $\gamma_{n}=\frac{n \pi}{b-a}+\mathrm{O}\left(\frac{1}{n}\right)$ are roots of $f\left(\gamma_{n}\right)=0$, provided that

(1) $f(\gamma)$ is an even function

(2) $f(0)=C$

(3) $f^{\prime}(0)=0$.

A careful analysis of these restrictions will show that they are satisfied, if $c=\left(b^{2}-a^{2}\right) /(\pi a b)$. Thus, we can now write

$$
\begin{aligned}
& f(\gamma)=\left[\frac{b^{2}-a^{2}}{\pi a b}\right]_{1}^{\infty}\left(1-\frac{k^{2}}{\gamma_{n}^{2}}+\frac{\zeta^{2}}{\gamma_{n}^{2}}\right) \\
& f(\gamma)=\frac{b^{2}-a^{2}}{\pi a b} \prod_{1}^{\infty}\left(\sqrt{1-\frac{k^{2}}{\gamma_{n}^{2}}}+\frac{i \zeta}{\gamma_{n}}\right) e^{-\frac{i \zeta(b-a)}{n \pi}} \prod_{1}^{\infty}\left(\sqrt{1-\frac{k^{2}}{\gamma_{n}^{2}}}-\frac{i \zeta}{\gamma_{n}}\right) e^{+\frac{i \zeta(b-a)}{n \pi}}
\end{aligned}
$$

where $e^{ \pm \frac{i \zeta(b-a)}{n \pi}}$ are inserted as absolute convergence factors, without which the infinite products are only conditionally convergent [12]. Similarly, if we expand

$$
J_{n}(z)=\frac{(z / 2)^{n}}{\Gamma(n+1)} \prod_{n=1}^{\infty}\left\{1-\frac{z^{2}}{x_{n}^{2}}\right\},
$$


where $J_{n}\left(x_{n}\right)=0$, then from $(56)$

$$
\begin{aligned}
J_{1}(\gamma a) & =\frac{a \gamma}{2} \Pi_{1}^{\infty}\left(1-\frac{(\gamma a)^{2}}{x_{n}^{2}}\right) \\
& =\frac{a \sqrt{k^{2}-\zeta^{2}}}{2} \Pi_{1}^{\infty}\left(\sqrt{1-\frac{k^{2} a^{2}}{x_{n}^{2}}}+\frac{i \zeta a}{x_{n}}\right) e^{-\left(\frac{i \zeta a}{n \pi}\right)} \prod_{1}^{\infty}\left(\sqrt{1-\frac{k^{2} a^{2}}{x_{n}^{2}}}-\frac{i \zeta a}{x_{n}}\right) e^{+\left(\frac{i \zeta a}{n \pi}\right)}
\end{aligned}
$$

and

$$
J_{1}(\gamma b)=\frac{b \sqrt{k^{2}-\zeta^{2}}}{2} \prod_{1}^{\infty}\left(\sqrt{1-\frac{k^{2} b^{2}}{x_{n}^{2}}}+\frac{i \zeta b}{x_{n}}\right) e^{-\frac{i \zeta b}{n \pi}} \prod_{1}^{\infty}\left(\sqrt{1-\frac{k^{2} b^{2}}{x_{n}^{2}}}-\frac{i \zeta b}{x_{n}}\right) e^{+\frac{i \zeta b}{n \pi}}
$$

Hence, we may have

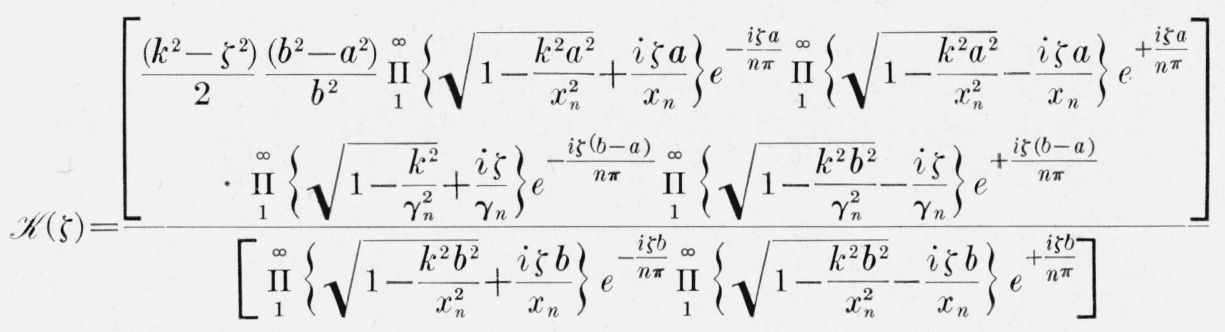

(c) Now, if we define, as shown in (58),

$$
\mathscr{K}(\zeta)=\frac{K_{-}(\zeta)}{K_{+}(\zeta)}
$$

where $K_{-}(\zeta)$ is regular and not zero in the lower half place, $\eta<0$, and $K_{+}(\zeta)$ is regular and not zero in the upper half plane, $\eta>-e_{1}>-\mathrm{K}_{1}$, our transform equation (47) becomes (51) which satisfies the requirements of the Liouville theorem because $\mathscr{V}(\zeta) K_{+}(\zeta)$ is regular for $\eta>-e_{1}$, and $\mathscr{K}(\zeta) K_{-}(\zeta)$ is regular for $\eta<0$ and the strip $0>\eta>-e_{1}>\mathbf{K}_{1}$ preserves the continuity.

We must now study the growth order of both members of (51) as $|\zeta| \rightarrow \infty$. Thus for $\mathscr{V}(\zeta)$ and $\mathscr{H}(\zeta)$, we examine the transforms

$$
\begin{aligned}
& \mathscr{V}(\zeta)=\int_{-\infty}^{0} e^{-i \zeta z} V(z) d z \\
& \mathscr{H}(\zeta)=\int_{0}^{\infty} e^{-i \zeta z} H(z) d z .
\end{aligned}
$$

The behavior of $V(z)=\left[\frac{\partial \phi}{\partial r}(r, z)\right]_{r=a,}$ as $z \rightarrow 0$, can be obtained by examining $\phi$ in the region of the sharp edge (zero thickness). In this region, where the values of $z$ are very much smaller than a wave length, $\phi$ satisfies Laplace's equation which can be solved by static methods. This is done by transforming the potential field about the sharp edge to the uniform region in the upper half plane with the aid of Schwarz-Christoffel [13] transformation, $W^{2}=2 z$. From this transformation, $W \sim z^{1 / 2}$ and the field which corresponds to $V(z)$ as $z \rightarrow 0$ at the sharp edge is $|d W / d z| \sim 2 z^{-1 / 2}$. Therefore, $V(z)$ which is related to the component of the particle velocity along the cylindrical radius at the inner pipe has an integrable singularity of the form $z^{-1 / 2}$ and

$$
\mathscr{V}(\zeta) \sim \int_{-\infty}^{0} \frac{e^{-i \zeta z}}{z^{1 / 2}} d z \sim(i \zeta)^{-1 / 2} \text { as }|\zeta| \rightarrow \infty \text { for } \eta>-e_{1}
$$


Also from the above transformation, we see that $H(z)$ which behaves as the velocity potential itself can be given by $H(z) \sim z^{1 / 2}$ as $z \rightarrow 0$ and

$$
\mathscr{H}(\zeta) \sim \int_{0}^{\infty} e^{-i \zeta z}\left(z^{1 / 2}\right) d z \sim \frac{1}{(i \zeta)^{3 / 2}} \text { as }|\zeta| \rightarrow \infty \text { for } \eta<0 .
$$

Thus, from a consideration of the physical nature of $V$ and $H$, we find that $\mathscr{H}$ and $\mathscr{V}$ behave in a purely algebraic manner as $|\zeta| \rightarrow \infty$. Therefore, the acceptable results of the decomposition of the kernel must behave in a similar fashion to permit the integral function to be bounded.

To properly decompose the kernel, we choose

$$
K_{+}(\zeta)=\frac{\prod_{1}^{\infty}\left[\sqrt{1-\frac{k^{2} b^{2}}{x_{n}^{2}}}-\frac{i \zeta b}{x_{n}}\right] e^{+\frac{i \zeta b}{n \pi}} e^{\chi(\zeta)}}{\prod_{1}^{\infty}\left[\sqrt{1-\frac{k^{2} a^{2}}{x_{n}^{2}}}-\frac{i \zeta a}{x_{n}}\right] e^{+\frac{i \zeta a}{n \pi}} \prod_{1}^{\infty}\left[\sqrt{1-\frac{k^{2}}{\gamma_{n}^{2}}}-\frac{i \zeta}{\gamma_{n}}\right] e^{+\frac{i \zeta(b-a)}{n \pi}}},
$$

where again

$$
e^{+\left(\frac{i \zeta a}{n \pi}\right)}, e^{+\left(\frac{i \zeta b}{n \pi}\right)}, \text { and } e^{+\frac{i \zeta(b-a)}{n \pi}}
$$

are inserted in each case as absolute convergence factors and the arbitrary factor $e^{x(5)}$ is put in to remove the exponential growth and thereby insuring the algebraic behavior of the $K$ 's by the proper choice of $\chi(\zeta)$. This choice will be determined from the asymptotic form of $K_{-}(\zeta)$ [or $\left.K_{+}(\zeta)\right]$ as $|\zeta| \rightarrow \infty$ for $\eta<0$.

We then find that $K_{+}$has an infinite number of poles on the negative imaginary axis for

$$
\eta<-\sqrt{\mathrm{K}_{1}^{2}-\left(\frac{\pi}{b}\right)^{2}\left(\frac{5}{8}\right)} \equiv-e_{1}>-\mathrm{K}_{1}
$$

and zeros for $\eta \leq-\mathrm{K}_{1}$ and is therefore regular and not zero in the upper half plane $\eta>-e_{1}$. By removing (63) from (58), we have left

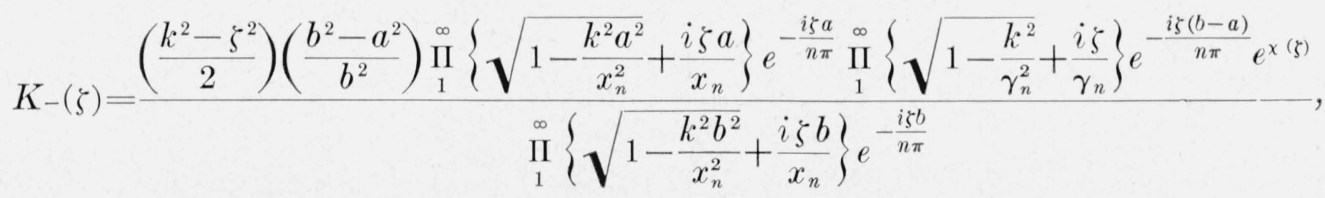

which is regular and not zero in the lower half plane, $\eta<0$, and has only simple poles and zeros in the upper half plane $\eta \geq 0$. We note, however, that $K_{-}$and $K_{+}$have a common region which is at least as large as $e_{1}$.

For the purpose of evaluating the asymptotic behaviors of $K_{-}$and $K_{+}$we may write $\gamma_{n} \sim n \pi /(b-a)$ and, since Schwinger [1] proved that the infinite products in (65) must be independent of $k$ as $|\zeta| \rightarrow \infty$, the square roots behave essentially as unity. Therefore, as $|\zeta| \rightarrow \infty$, we may use the Weirstrass [14] definition of the $\Gamma$ function and the Sterling asymptotic formula [15] for $\Gamma(\zeta)$ to write, as a typical example,

$$
\prod_{1}^{\infty}\left\{\sqrt{1-\frac{k^{2}}{\gamma_{n}^{2}}}+\frac{i \zeta}{\gamma_{n}}\right\} e^{-\frac{i \zeta(b-a)}{n \pi} \sim \frac{1}{e^{(C-1) x} x^{x+1 / 2} \sqrt{2 \pi}},}|\zeta| \rightarrow \infty, \quad \eta<0,
$$

where $x=i \zeta(b-a) / \pi$ and $C=$ Euler's constant. Similarly,

$$
\prod_{1}^{\infty}\left\{\sqrt{1-\frac{k^{2} a^{2}}{x_{n}^{2}}}+\frac{i \zeta a}{x_{n}}\right\} e^{-\frac{i \zeta a}{n \pi}} \sim \frac{\Gamma(5 / 4)}{e^{C y} e^{-\left(y+\frac{1}{4}\right)}\left(y+\frac{1}{4}\right)^{\frac{1}{4}} \sqrt{2 \pi\left(y+\frac{1}{4}\right)}}
$$


where $y=\frac{i \zeta a}{n \pi}$.

Thus as $|\zeta| \rightarrow 0$, we have

$$
K_{-}(\zeta)=\left(\frac{k^{2}-\zeta^{2}}{2}\right)\left(\frac{b^{2}-a^{2}}{b^{2}}\right) \frac{b^{\left(\frac{i \zeta b}{\pi}\right)} \sqrt{2 i \zeta b} e^{x(\zeta)}}{a^{\left(\frac{i \zeta a}{\pi}\right)} \sqrt{2 i \zeta a}(b-a)^{\left(\frac{i \zeta(b-a)}{\pi}\right)} \sqrt{2 i \zeta(b-a)}}
$$

which approaches $(i \zeta)^{3 / 2}$, provides that

$$
\chi(\zeta)=\frac{-i \zeta}{\pi}[b \ln b-a \ln a-(b-a) \ln (b-a)]
$$

to insure the algebraic behavior of both $K_{-}$and $K_{+}$. Likewise, we may show that

$$
K_{+}(\zeta) \rightarrow(-i \zeta)^{\frac{1}{2}} \text { or that } \mathscr{K}(\zeta) \rightarrow \zeta
$$

Collecting results we see that $\mathscr{V}(\zeta) K_{+}(\zeta)$ is bounded as $|\zeta| \rightarrow \infty$ for $\eta>-e_{1}$ and $\mathscr{H}(\zeta)$ $K_{-}(\zeta)$ is bounded as $|\zeta| \rightarrow \infty$ for $\eta<0$. Thus, we have shown that both sides of (51) define an analytic function which is regular everywhere including a common strip of width $e_{1}$ and is bounded at infinity. Therefore, by the statement of the Liouville theorem, (51) becomes

$$
\mathscr{V}(\zeta) K_{+}(\zeta)=\mathscr{H}(\zeta) K_{-}(\zeta)=C
$$

If we were interested in the difference of the velocity potential on either side of the inner pipe, we could now obtain it by evaluating

$$
\frac{1}{2 \pi i} \int_{B r} \frac{C e^{i \zeta z} d z}{K_{-}(\zeta)}
$$

where $\mathrm{Br}$ is an infinite contour within the strip of regularity $|\eta|<\mathrm{K}_{1}$. (d) We are now in a position to evaluate the Fourier integral form of (27), which we may write as

$$
\phi(r, z)=\frac{1}{2 \pi} \int_{i \sigma+\infty}^{i \sigma+\infty} d e^{i \zeta z} H(\zeta)\left[\frac{\partial \mathscr{G}}{\partial r^{\prime}}\left(r, r^{\prime}, \zeta\right)\right]_{r^{\prime}=a}
$$

and where we recall $\mathscr{G}$ exists in the strip $-K_{1}<\eta<K_{1}$ and $\mathscr{H}(\zeta)$ exists for $\eta<0$. Therefore the path of integration must be taken in the region $0>\eta>-K_{1}$.

If we concentrate for the moment on $r<r^{\prime}$, we have from (14)

and

$$
\left(\frac{\partial \mathscr{G}}{\partial r^{\prime}}\right)_{r^{\prime}=a}=\frac{\pi}{2} \gamma \frac{J_{0}(\gamma r)}{J_{1}(\gamma b)}\left[-J_{1}(\gamma a) N_{1}(\gamma b)+N_{1}(\gamma a) J_{1}(\gamma b)\right]
$$

$$
\left.\begin{array}{rl}
\mathscr{K}(\zeta) & =\frac{\pi}{2} \gamma^{2} \frac{J_{1}(\gamma a)}{J_{1}(\gamma b)}\left[J_{1}(\gamma a) N_{1}(\gamma b)-N_{1}(\gamma a) J_{1}(\gamma b)\right] \\
& =\frac{K_{-}(\zeta)}{K_{+}(\zeta)} .
\end{array}\right\}
$$

Therefore, if we form the ratio

$$
\frac{\left(\frac{\partial \mathscr{G}}{\partial r^{\prime}}\right)_{r^{\prime}=a}}{\mathscr{K}(\zeta)}=-\frac{J_{0}(\gamma r)}{\gamma J_{1}(\gamma a)}
$$

then

$$
\frac{\partial G}{\partial r^{\prime}}=-\frac{J_{0}(\gamma r)}{\gamma J_{1}(\gamma a)} \frac{K_{-}(\zeta)}{K_{+}(\zeta)}
$$


Now the integral in (71) becomes

$$
\frac{1}{2 \pi} \int_{i \sigma-\infty}^{i \sigma+\infty} d \zeta e^{i \zeta z} \frac{\mathscr{H}(\zeta) K_{-}(\zeta)}{K_{+}(\zeta)} \frac{\left[-J_{0}(\gamma r)\right]}{\left[J_{1}(\gamma a)\right]}
$$

which has simple poles at $\zeta= \pm k(\gamma=0)$ and $\zeta= \pm i \mathrm{~K}_{n}$.

The contributions at the poles on the imaginary axis vanish as $|z| \rightarrow \infty$ since they contain $e^{-K_{n}|z|}$. For $z<0$, we closed the path of integration by an infinite contour in the lower half plane. Since the poles enclosed are all on the imaginary axis, the contribution to the integral vanishes as $z \rightarrow-\infty$ and the result claimed in section $V$ is established. For $z>0$ and large, we use a contour in the upper half plane which encloses $\zeta= \pm k$ and (71) becomes

$$
\phi(r, z)=i D\left\{\frac{e^{-i k z}}{2 k K_{+}(-k)}-\frac{e^{i k z}}{2 k K_{+}(k)}\right\}+\frac{1}{2}\left[I_{1}(z)+\frac{Z_{2}}{Z_{1}} I_{2}(z)+\frac{Z_{3}}{Z_{1}} I_{3}(z)\right]
$$

since only the residues at the poles on the real axis contribute as $z \rightarrow \infty$. Likewise, if we consider $r>r^{\prime}$, it can easily be verified that the result is the same as (77) with a different constant $D$.

\section{Expression "d" in Terms of Infinite Series}

In (77), we have evaluated the integral representation (27) from its Fourier Integral form (71) and we can now obtain the far fields to the right. For now, as $z \rightarrow \infty, \phi(r, z) \rightarrow I_{3}(z)$ in region 3 and $I_{2}(z)$ in region 2 . In terms of the parameters at $z=0$, we have similar results in regions 2 or 3 with different constants $A$ namely

$$
\begin{aligned}
I_{2,3} \cos k z-i Y_{2,3} V_{2,3} \sin k z & =A_{2,3}\left[\frac{e^{i k z}}{K_{+}(-k)}-\frac{e^{i k z}}{K_{+}(k)}\right]+\frac{\cos k z}{2}\left[I_{1}+\frac{Z_{2}}{Z_{1}} I_{2}+\frac{Z_{3}}{Z_{1}} I_{3}\right] \\
& -i \frac{\sin k z}{2}\left[V_{3} Y_{3}+V_{2} Y_{2}-V_{1} Y_{1}\right] .
\end{aligned}
$$

If we, again, equate coefficients of sin and cos, we have the far fields results extrapolated from the right as

$$
\left[I_{2,3}-\frac{1}{2}\left(I_{1}+\frac{Z_{2}}{Z_{1}} I_{2}+\frac{Z_{3}}{Z_{1}} I_{3}\right)\right]=A_{2,3}\left[\frac{1}{K_{+}(-k)}-\frac{1}{K_{+}(k)}\right]
$$

and

$$
\left[-i V_{2,3} Y_{2,3}+\frac{1}{2}\left(V_{3} Y_{1}+V_{2} Y_{1}-V_{1} Y_{1}\right)\right]=A_{2,3}\left[\frac{-i}{K_{-}(-k)}-\frac{i}{K_{+}(k)}\right] .
$$

Recalling that the results from the left gave the circuit equations (32) and (33), we apply them to the above and find that

and

$$
\left[I_{2,3}-I_{1}\right]=A_{2,3}\left[\frac{1}{K_{+}(-k)}-\frac{1}{K_{+}(k)}\right]
$$

$$
-i\left(V_{2,3} Y_{2,3}+V_{1} Y_{1}\right)=-i A_{2,3}\left[\frac{1}{K_{+}(-k)}+\frac{1}{K_{+}(k)}\right]
$$

By simply dividing these two equations, we see from (40), that the left side is merely the one essential circuit parameter $b$ discussed in section $\mathrm{V}$ and hence with (46), we have

$$
B=i\left[\frac{K_{+}(k)-K_{+}(-k)}{K_{+}(k)+K_{+}(-k)}\right]=\tan (k d),
$$


where the reciprocal of $K_{+}(k)$ is

$$
\frac{1}{K_{+}(k)}=\frac{\prod_{1}^{\infty}\left\{\sqrt{\left.1-\frac{k^{2} a^{2}}{x_{n}^{2}}-\frac{i k a}{x_{n}}\right\} e^{\frac{i k a}{n \pi}} \prod_{1}^{\infty}\left\{\sqrt{1-\frac{k^{2}}{\gamma_{n}^{2}}}-\frac{i k}{\gamma_{n}}\right\} e^{\frac{i k(b-a)}{n \pi}}}\right.}{\prod_{1}^{\infty}\left\{\sqrt{1-\frac{k^{2} b^{2}}{x_{n}^{2}}}-\frac{i k b}{x_{n}}\right\} e^{\frac{i k b}{n \pi}} e^{x(k)}} .
$$

By writing the bracketed terms in (84) as exponentials, for these are of the form $\cos \theta-i \sin \theta$, we see that (83) becomes

$\frac{\pi d}{b}=\left[\ln \alpha+\left(\frac{1}{\alpha}-1\right) \ln (\alpha-1)\right]-\frac{1}{\eta} \sum_{n=1}^{\infty}\left[\sin ^{-1}\left(\frac{\eta \pi}{\alpha x_{n}}\right)-\left(\frac{\eta}{\alpha n}\right)-\frac{1}{\eta} \sum_{n=1}^{\infty}\left[\sin ^{-1}\left(\frac{\eta \pi}{\alpha y_{n}}\right)-\left(\frac{\eta(\alpha-1)}{\alpha n}\right)\right]+\frac{1}{\eta} \sum_{n=1}^{\infty}\left[\sin ^{-1}\left(\frac{\eta \pi}{x_{n}}\right)-\left(\frac{\eta}{n}\right)\right]\right.$

where $y_{n}$ are the roots of

$$
J_{1}\left(y_{n}\right) N_{1}\left(\alpha y_{n}\right)-J_{1}\left(\alpha y_{n}\right) N_{1}\left(y_{n}\right)=0
$$

and $x_{n}$ are roots of $J_{1}\left(x_{n}\right)=0$. For ease in computation, we have made the following parameter changes:

1. Let $\alpha=b / a$.

2. From our initial restriction on the driving wavelength, let $k=\eta \pi / b$, where $0 \leq \eta \leq 1$.

3. Let $\left(\gamma_{n} a\right)=y_{n}$.

Since we now have an analytic expression for $d$, it is of considerable interest to determine $d$ as a function of frequency and the radii of the tubes. To evaluate $d$ numerically, mustwe find some way to get an accurate result for the above infinite series which is of the form

$$
\sum_{n=1}^{\infty}\left\{\left[\sin ^{-1}\left(X_{n}\right)-Y_{n}\right]\right\}=\sum_{n=1}^{\infty}\left\{\left[\sin ^{-1}\left(X_{n}\right)-\left(X_{n}\right)+\left(X_{n}-Y_{n}\right)\right\} .\right.
$$

The first two terms can be written as

$$
\sum_{n=1}^{N}\left[\sin ^{-1}\left(X_{n}\right)-X_{n}\right]+R_{n}
$$

where $N$ is some arbitrary finite number. Thus by calculating $N$ simple values and bounding the remainder, we will be able to evaluate these sums Writing $\sin ^{-1}$ as an infinite series,

$$
R_{n}=\sum_{N+1}^{\infty}\left[\sin ^{-1}\left(X_{n}\right)-X_{n}\right]=\sum_{N+1}^{\infty}\left[+\frac{1}{6}\left(X_{n}\right)^{3}+\frac{3}{40}\left(X_{n}\right)^{5}+\frac{5}{112}\left(X_{n}\right)^{7}+\ldots\right]
$$

we have a series which converges rapidly for $X_{n}<1$.

If we now define $U_{N+1}$ as

$$
U_{n+1}=\frac{\sin ^{-1}\left(X_{n+1}\right)-X_{n+1}}{\left(X_{n+1}\right)^{3}} \sim \frac{1}{6}
$$

we see that the numerator is the largest term in the remainder and that $U_{N+1}$ approaches $1 / 6$ as $N$ grows large. Now from the series expansion, (89), it can be easily verified that

$$
\frac{\sin ^{-1}\left(X_{N+1}\right)-X_{N+1}}{\left(X_{N+1}\right)^{3}} \geq \frac{\sin ^{-1}\left(X_{N+1}\right)-X_{N+1}}{\left(X_{N+1}\right)^{3}}
$$

for $i=1,2,3$, since $X_{N+1} \geq X_{N+i}$. 
Thus from (90) and (91)

$$
U_{N+1}\left(X_{N+1}\right)^{3}>\sin ^{-1}\left(X_{N+i}\right)-X_{N+i}
$$

which means that

$$
R_{n}<U_{N+1} \sum_{N+1}^{\infty}\left(X_{n}\right)^{3}
$$

Also from the series, we have, by dropping higher order terms,

$$
R_{n}>\sum_{N+1}^{\infty} \frac{1}{6}\left(X_{n}\right)^{3}
$$

which together with (93) bounds $R_{n}$ as

$$
\frac{1}{6} \sum_{N+1}^{\infty}\left(X_{n}\right)^{3}<R_{n}<U_{N+1} \sum_{N+1}^{\infty}\left(X_{n}\right)^{3} .
$$

Thus, we can easily determine a numerical value for $R_{n}$. The remaining sums of (87) may be easily obtained since $\lim _{n \rightarrow \infty}\left(X_{n}\right)=Y_{n}$.

The infinite sums in (85) were evaluated by the computation laboratory of the Institute for Numerical Analysis, National Bureau of Standards, for $\eta$, the frequency parameter, assuming the values of $1, .8, .6, .4, .2$ and 0 . Although $\eta=0$ has no physical meaning in the present acoustic problem, for completeness the mathematical $\lim \pi d / b$ was evaluated to show that $d$ is a smooth and continuous function of $\eta$ for $\eta=0$. Thus since

equation (85) becomes

$$
\lim _{\eta \rightarrow 0} \sin ^{-1} \frac{\eta}{C}=\frac{1}{C}
$$

$$
\frac{\pi d}{b}=\left[\ln \alpha+\left(\frac{1}{\alpha}-1\right) \ln (\alpha-1)\right]-\sum_{n=1}^{\infty}\left[\frac{\pi}{\alpha x_{n}}-\frac{\pi}{x_{n}}+\frac{\pi}{\alpha y_{n}}\right]
$$

as $\eta \rightarrow 0$. The values of $\alpha$ used were $1.05,1.2,1.5,2,3,5$, and 10 , while $\alpha=1.0$ and $\infty$ would mean that there is no inner guide; consequently $d=0$. The computations were intended to be accurate to four decimal places, but with the methods outlined greater accuracy could have

\begin{tabular}{|c|c|c|c|c|c|c|c|c|}
\hline \multicolumn{8}{|c|}{$y_{n}$} & \multirow{2}{*}{$x_{n}$} \\
\hline$n$ & 1.05 & 1. 2 & 1.5 & 2.0 & 3.0 & 5.0 & 10.0 & \\
\hline $\begin{array}{r}1 \\
2 \\
3 \\
4 \\
5 \\
6 \\
7 \\
8 \\
9 \\
10 \\
11 \\
12 \\
13 \\
14 \\
15\end{array}$ & $\begin{array}{r}62.837 \\
125.666 \\
188.497 \\
251.329 \\
314.160 \\
376.922 \\
439.824\end{array}$ & $\begin{array}{r}15.728 \\
31.426 \\
47.131 \\
62.837 \\
78.544 \\
94.251 \\
109.958 \\
125.666\end{array}$ & $\begin{array}{l}6.3218 \\
12.586 \\
18.863 \\
25.143 \\
31.424 \\
37.706 \\
43.988 \\
50.270 \\
56.553 \\
62.836\end{array}$ & $\begin{array}{c}3.1966 \\
6.3124 \\
9.4446 \\
12.581 \\
15.720 \\
18.860 \\
21.999 \\
25.141 \\
28.281 \\
31.422 \\
34.563\end{array}$ & $\begin{array}{c}1.6356 \\
3.1789 \\
4.7381 \\
6.3028 \\
7.8696 \\
9.4375 \\
11.007 \\
12.576 \\
14.146 \\
15.716 \\
17.286\end{array}$ & $\begin{array}{r}\text { 0. } 84715 \\
1.6111 \\
2.3853 \\
3.1642 \\
3.9454 \\
4.7279 \\
5.5112 \\
6.2950 \\
7.0791 \\
7.8635 \\
8.6480 \\
9.4327 \\
10.217\end{array}$ & $\begin{array}{l}0.39409 \\
.73306 \\
1.9748 \\
1.4189 \\
1.7643 \\
2.1107 \\
2.4578 \\
2.8052 \\
3.1529 \\
3.5010 \\
3.8492 \\
4.1975 \\
4.5459 \\
4.8944 \\
5.2430\end{array}$ & $\begin{array}{r}3.8317 \\
7.0156 \\
10.1735 \\
13.324 \\
16.471 \\
19.616 \\
22.760 \\
25.904 \\
29.047 \\
32.190 \\
35.332 \\
38.475 \\
41.617 \\
44.759 \\
47.902\end{array}$ \\
\hline
\end{tabular}
been obtained. The eigenvalues $y_{n}$ (table 1) for small $n$ were obtained from Muskat et al [16] with corrections given by Miller [17] and for large $n$ were computed with the aid of a formula give by McMahon [18]. The results of (85) are exhibited in tables 1 and 2 and in figures 7 and 8 .

TABLE 1 


\begin{tabular}{|c|c|c|c|r|r|}
\hline \multicolumn{2}{|c|}{$\left[\frac{\pi d}{b}\right]$} & \\
\hline \\
\hline
\end{tabular}

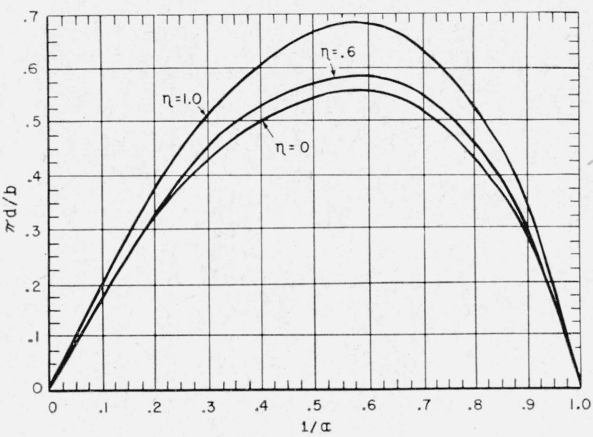

Figure 8.

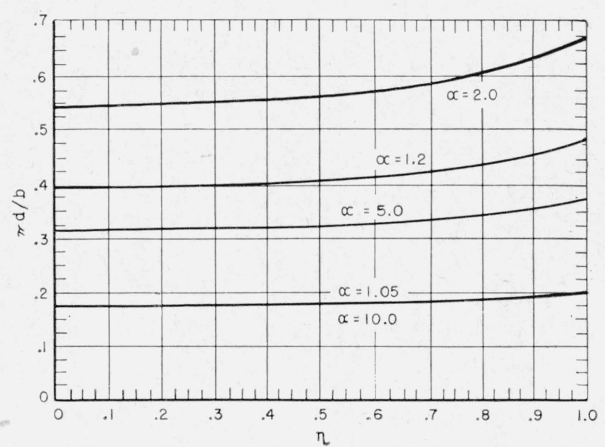

Figure 9 .

\section{Conclusion}

In (85), we have obtained a rigorous and explicit solution for the parameter $d$. An approximate solution of the above problem was first reported by J. W. Miles [7], who showed by using impedance concepts developed in earlier papers and the variational principle of Schwinger's that the equivalent circuit elements can be related to the analogous change of cross section. Thus by considering the bifurcation as a mere change of cross section, he obtained his equation (53) which contained only the leading term of an infinite series. A comparison of the results given in table 2 and the values given by Miles indicates, that for this problem, this type of approximation is very poor. Attempts have been made to improve this method of approximation by considering two terms of the infinite series and only slightly better results were obtained. An explanation of these discrepancies between the exact results given here and the approximations is to be given by Miles [19].

The author is deeply indebted to Professor Alfredo Baños, Jr., for the guidance that he has so graciously given throughout all phases of this research and to Professors Baños and David S. Saxon for the suggestion of this problem, as well as the benefit of their experience in the Radiation Laboratory of Massachusetts Institute of Technology.

\section{References}

[1] J. Schwinger, The theory of guided waves, Radiation Laboratory lectures. Unpublished. Communicated to the author by Professors Alfred Baños, Jr., and David S. Saxon of UCLA.
[2] R. E. A. C. Paley and N. Wiener, The Fourier transform in the complex domain, Am. Math. Soc. Colloquium Publication (1934) Vol. XIX. E. Reissner, J. Math. Phys. MIT (20) 5, 219 (1941). 
[3] Levine and Schwinger, Phys. Rev. 73, No. 4, 383 to 406 (Feb. 1948).

[4] Courant and Hilbert, Methoden der Mathematischen Physik, I, Sec. Ed., p. 303 to 304 (Julius Springer, 1931).

[5] J. W. Miles, J. Acous. Soc. Am. 17, Part I, 259 to 272 (1946).

[6] Everett, Communication Engineering, p. 52 (McGrawHill Book Co., Inc., New York, N. Y., 1937).

[7] J. W. Miles, J. Acous. Soc. Am 19, No. 4, 579 to 584 (July 1947).

[8] Whittaker and Watson, A course of modern analysis, p. 105 (Am. Ed., Macmillan Co., New York, N. Y., 1945).

[9] Placzek and Seidel, Phys. Rev. 72, No. 7, p. 550 to 555 (Oct. 1947).

[10] J. F. Carlson and A. E. Heins, Quart. Applied Math., Part I, 4, No. 7, 313 to 329; Part II, 5, No. 1, 82 to 88 (1947).
[11] Whittaker and Watson, Modern analysis, p. 137 (Macmillan Co., New York, N. Y., 1947).

[12] Watson.

[13] Smythe, Static and dynamic electricity, p. 82, first ed. (McGraw-Hill Book Co., Inc., New York, N. Y., 1939).

[14] Whittaker and Watson, Modern analysis, p. 236 (Macmillan Co., New York, N. Y., 1947).

[15] Whittaker and Watson, Modern analysis, p. 253 (Macmillan Co., New York, N. Y., 1947).

[16] M. Muskat, F. Morgan, and M. W. Meres, J. App. Phys. 11, 212 (1940).

[17] Bateman and Archibald, MTAC v. 1., 7, 325 (1944). See comments by J. C. P. Miller.

[18] J. MeMahon, Annals Math. 9, 23 to 30 (1895).

[19] J. W. Miles, J. Acous. Soc. Am. 22, No. 1, 59 to 60 (1950).

Los Angeles, November 2. 1949. 